\title{
ESTADO NUTRICIONAL Y CONDICIÓN FÍSICA DE FUTBOLISTAS ADOLESCENTES LUEGO DEL CONSUMO DE HARINA DE PESCADO COMO COMPLEMENTO NUTRICIONAL
}

\author{
Roberto Accinelli-Tanaka ${ }^{1,2, a}$, Lidia López-Oropeza ${ }^{2, b}$
}

\author{
RESUMEN
}

\begin{abstract}
El objetivo del estudio fue determinar los cambios en los parámetros nutricionales y condición física en adolescentes deportistas luego de consumir harina de pescado como complemento nutricional. Para ello, se realizó un estudio cuasiexperimental, ciego para los investigadores, en 100 adolescentes futbolistas, divididos en dos grupos homogéneos en todos los parámetros de estudio. Se le brindó harina de pescado durante cuatro meses a uno de los grupos. Luego de evaluar el estado nutricional y la condición física, antes y después de la intervención, no se encontraron cambios en el estado nutricional, ni antropométricos ni de laboratorio, tampoco en la condición física; pero sí en los niveles de hemoglobina y hematocrito entre los que consumieron la harina de pescado y el grupo control. En conclusión, el consumo de harina de pescado no se tradujo en cambios en el estado nutricional ni en la condición física de adolescentes deportistas.
\end{abstract}

Palabras clave: Harina de pescado; Adolescente; Fútbol; Deportes; Estado nutricional; Resistencia física (Fuente: DeCS BIREME)

\section{NUTRITIONAL STATUS AND PHYSICAL CONDITION OF ADOLESCENT FOOTBALL PLAYERS AFTER CONSUMING FISHMEAL AS A NUTRITIONAL COMPLEMENT}

\begin{abstract}
The objective of the study is to identify the changes in the nutritional parameters and the physical condition of teenage players after eating fishmeal as a nutritional complement. For this purpose, a quasi-experimental study, blinded for investigators, was conducted, involving 100 teenage football players, divided in two groups, homogeneous in terms of all study parameters, one of which received fishmeal for four months. After evaluating the nutritional status and physical condition, before and after the intervention, no change was found in the nutritional and anthropometric status or laboratory results, or in the physical condition. However, those who received fishmeal did report a change in their hemoglobin and hematocrit levelsin comparison to the control group. In conclusion, the consumption of fishmeal did not lead to changes in the nutritional status or the physical condition of teenage football players.
\end{abstract}

Key words: Fish flour; Adolescent; Soccer; Sports; Nutritional status; Physical endurance [source: MeSH NLM].

\section{INTRODUCCIÓN}

El requerimiento energético nutricional en niños y adolescentes es mayor que en el adulto debido a la alta demanda energética requerida para el crecimiento físico y desarrollo ${ }^{(1)}$. En aquellos que practican regularmente algún tipo de deporte este requerimiento se incrementa aun más para cubrir la demanda adicional producto de la actividad física ${ }^{(2)}$. La Organización de las Naciones Unidad para la Alimentación y Agricultura (FAO) y la Organización Mundial de la Salud (OMS) recomiendan $2839 \mathrm{kcal} / \mathrm{d}$ en promedio, para varones de 10 a 18 años; este valor se incrementa en $14,9 \%$ en aquellos adolescentes que realicen actividades físicas intensas (3). En el Perú, el Centro Nacional de Alimentación y Nutrición ha estimado que los adolescentes varones de 12 a 17 años procedentes de áreas urbanas necesitan en promedio $2565 \mathrm{kcal} / \mathrm{d}$, en tanto que aquellos que realizan actividades físicas intensas requieren $2895 \mathrm{kcal} / \mathrm{d}{ }^{(4)}$.

Parte de este requerimiento energético debe ser cubierto por una cantidad apropiada de proteínas, por lo

\footnotetext{
Instituto de Investigaciones de la Altura, Universidad Peruana Cayetano Heredia. Lima, Perú.

Servicio de Neumología, Hospital Nacional Cayetano Heredia. Lima, Perú.

Médico neumólogo magister en Salud Pública, ${ }^{\mathrm{b}}$ médico cirujano.

Recibido: 27-09-12 Aprobado: 23-01-13
}

Citar como: Accinelli-Tanaka R, López-Oropeza L. Estado nutricional y condición física de futbolistas adolescentes luego del consumo de harina de pescado como complemento nutricional. Rev Peru Med Exp Salud Publica. 2013;30(1):49-53. 
que la FAO recomienda que los adolescentes varones de 10 a 18 años deban consumir $0,95 \mathrm{~g}$ de proteínas por kilo de peso por día ${ }^{(5)}$. En tanto que los adolescentes deportistas necesitan entre 1,2 y 1,9 g de proteínas por kilo de peso por día ${ }^{(6,7)}$, ello debido a que la mayor oxidación de aminoácidos producida durante el ejercicio, sobre todo en los de resistencia y fuerza, determinan un mayor requerimiento de proteínas ${ }^{(2,8)}$.

Se hace entonces necesario incrementar en la dieta de los adolescentes deportistas el aporte proteico que proporcione el sustrato necesario para cubrir la demanda generada por el crecimiento físico y el ejercicio. El Perú es el principal exportador de harina de pescado del mundo ${ }^{(9)}$, la cual se elabora fundamentalmente a base de anchoveta. Esta harina constituye una fuente importante y económica de proteínas de origen animal que podría ser empleada para cubrir el mayor requerimiento proteico en deportistas. Es por ello que el objetivo de este estudio fue determinar los cambios que se producen en los parámetros nutricionales y en la condición física de futbolistas adolescentes luego de consumir harina de anchoveta.

\section{EL ESTUDIO}

Se realizó un estudio cuasi-experimental, ciego simple para los investigadores, en un grupo de adolescentes futbolistas amateurs del Club Cantolao, sede en el Callao, durante los meses de abril a agosto de 2010. Se realizó un muestreo no probabilístico que incluyó a cien adolescentes, el criterio de inclusión fue el tener de 9 a 18 años. Se excluyeron a aquellos adolescentes que mostraron antecedentes de alergia a pescado; enfermedad hepática, renal o cardiovascular; diabetes mellitus, o que estuvieran recibiendo alguna medicación.

Para la intervención se utilizó harina de pescado producida por Pesquera Capricornio S.A., la cual contiene por cada $100 \mathrm{~g}: 69,7 \%$ de proteínas; $10,0 \%$ de grasas; $7,5 \%$ de ácidos grasos libres; histamina; antioxidantes (decanox); dioxina; enterobacterias $(<300 \mu \mathrm{fc} / \mathrm{g})$, y otros. Todos en cantidades aptas para el consumo humano según los análisis realizados en el Laboratorio SGS del Perú S.A.C y en el de Microbac Laboratories Inc. (EE. UU.) el cual es supervisado por la Food and Drug Administration (FDA) de EE. UU.

Se dividió a los participantes en dos grupos de cincuenta adolescentes. De lunes a viernes, durante el almuerzo, los adolescentes del grupo de intervención recibieron como único aporte de proteínas de origen animal $50 \mathrm{~g}$ de harina de pescado en forma de fideos, panes, biscochos y pizzas; los fines de semana $20 \mathrm{~g}$ de la harina en forma de galletas. La preparación del almuerzo y las galletas fue realizada por un cocinero profesional. La supervisión de la ingesta así como el control de las reacciones adversas fue realizada por una persona capacitada. El grupo control continuó con su alimentación habitual en casa. En ninguno de los grupos se realizó la medición basal del aporte proteico y calórico diario.

La actividad física consistió en ejercicios físicos, tácticos, técnicos y partidos de fútbol por un periodo de noventa minutos diarios.

La antropometría se realizó con el adolescente descalzo y vistiendo camiseta y short deportivo. Se midió el peso y la talla, para ello se empleó una balanza Camry ${ }^{\circledR}$ calibrada de $0-120 \mathrm{~kg}$ con una precisión de $100 \mathrm{~g}$, y un tallímetro inextensible, transportable, rígido, con un intervalo de 0-184 cm y una precisión de 0,1 cm. El índice de masa corporal (IMC) se calculó dividiendo el peso entre la talla al cuadrado. Para la valoración de los parámetros bioquímicos del estado nutricional se determinaron los niveles de proteínas totales, albúmina, hemoglobina y hematocrito. Para la valoración de la condición física se empleó la prueba de Cooper en la que, manteniendo una velocidad constante y uniformemente acelerada sobre un terreno plano y homogéneo, cada participante recorre la mayor distancia posible en doce minutos. La condición física, en varones menores de 30 años se clasifica como muy mala (< 1,6 km); mala (1,6 a < 2,2 km); regular (2,2 a < 2,4 km); buena (2,4 a 2,8 km), y excelente (> 2,8 km). Todas estas mediciones se realizaron al inicio del estudio y luego de cuatro meses de ingesta de la proteína de anchoveta.

El análisis estadístico incluyó una descripción basal de ambos grupos, determinando promedio y desviación estándar para las variables continuas, y frecuencia para las variables categóricas. La normalidad de las variables se evaluó con la prueba de Shapiro Swilk. Para el análisis de las variables categóricas se usó la T de Student para muestras relacionadas y la $U$ de MannWhitney Willcoxon, según correspondiese. Para las variables continuas se empleó la prueba T de Student para muestras independientes.

El estudio fue revisado y aprobado por el Comité Institucional de Ética de la Universidad Peruana Cayetano Heredia (código 56755). Los adolescentes que aceptaron participar en el estudio firmaron un asentimiento informado previo consentimiento de los padres.

\section{HALLAZGOS}

Se incluyó cien adolescentes deportistas de sexo masculino, la media de la edad fue de de 15,2 $\pm 2,0$ años. La medición basal demostró que los dos 
Tabla 1. Parámetros nutricionales y de condición física en adolescentes deportistas en complementación nutricional con harina de pescado.

\begin{tabular}{|c|c|c|c|c|c|c|}
\hline & \multicolumn{3}{|c|}{ Grupo de intervención } & \multicolumn{3}{|c|}{ Grupo control } \\
\hline & $\begin{array}{c}\text { Antes } \\
(n=49)^{*} \\
\text { Media } \pm \mathrm{DE} \\
\end{array}$ & $\begin{array}{c}\text { Después } \\
(n=46) \\
\text { Media } \pm \mathrm{DE}\end{array}$ & $p$ & $\begin{array}{c}\text { Antes } \\
(n=49)^{\star} \\
\text { Media } \pm \mathrm{DE} \\
\end{array}$ & $\begin{array}{c}\text { Después } \\
(n=31) \\
\text { Media } \pm \mathrm{DE}\end{array}$ & $p$ \\
\hline \multicolumn{7}{|l|}{ Pruebas hematológicas } \\
\hline Hemoglobina (g/dL) & $14,2 \pm 0,9$ & $14,5 \pm 1,1$ & 0,003 & $14,7 \pm 1,1$ & $15,0 \pm 1,1$ & 0,027 \\
\hline Hematocrito (\%) & $43,0 \pm 2,8$ & $44,1 \pm 3,2$ & 0,002 & $44,5 \pm 2,7$ & $45,0 \pm 2,9$ & 0,264 \\
\hline \multicolumn{7}{|l|}{ Pruebas bioquímicas } \\
\hline Proteínas totales (g/dL) & $7,0 \pm 0,3$ & $7,1 \pm 0,4$ & 0,365 & $7,1 \pm 0,3$ & $7,0 \pm 0,5$ & 0,112 \\
\hline Albúmina $(\mathrm{g} / \mathrm{dL})$ & $3,9 \pm 0,1$ & $3,9 \pm 0,3$ & 0,446 & $4,0 \pm 0,1$ & $4,0 \pm 0,3$ & 0,881 \\
\hline \multicolumn{7}{|l|}{ Medidas antropométricas } \\
\hline $\mathrm{IMC}\left(\mathrm{kg} / \mathrm{m}^{2}\right)$ & $20,9 \pm 2,3$ & $20,9 \pm 2,4$ & 0,062 & $21,8 \pm 2,2$ & $21,9 \pm 2,3$ & 0,607 \\
\hline Prueba de Cooper $(\mathrm{km})$ & $2,6 \pm 0,2$ & $2,4 \pm 0,4^{\dagger}$ & $<0,001$ & $2,6 \pm 0,3$ & $2,4 \pm 0,3^{\dagger}$ & 0,024 \\
\hline
\end{tabular}

$\mathrm{DE}$ : desviación estándar; IMC: índice de masa corporal.

"Se registró una muestra coagulada en el grupo control y un participante no aceptó la toma de muestra en el grupo de intervención.

† diez adolescentes del grupo de intervención y ocho del control no aceptaron realizar la prueba de Cooper.

grupos diferían en edad e IMC; las medias fueron 14,4 y 15,9 años $(p<0,001)$ y 20,9 y $22,2 \mathrm{~kg} / \mathrm{m}^{2}$ para el grupo intervención y control respectivamente, diferían además en las pruebas hematológicas en hemoglobina y hematocrito; las medias fueron 14,2 y $14,7 \mathrm{mg} / \mathrm{dl}(\mathrm{p}=0,03)$ y 43,1 y $44,5 \%$ ( $p=0,014)$. No se encontró diferencia en los demás parámetros evaluados (proteínas $[p=0,24]$; albúmina $[p=0,52]$, y prueba de Cooper $[p=0,42])$. Los resultados de las pruebas de sangre se encontraron dentro de límites normales en todos los casos. El 85\% del grupo intervención y el $81 \%$ del grupo control tuvieron una condición física buena o excelente.

A la segunda medición solo acudieron 46 adolescentes del grupo de intervención y 31 del grupo control; adicionalmente, diez adolescentes del grupo de intervención y ocho del grupo de control no aceptaron realizar la prueba de Cooper, lo cual implicó una pérdida global del $23 \%$ de participantes para los exámenes de sangre (8\% del grupo de intervención y $38 \%$ del grupo control) y del $41 \%$ para la prueba de Cooper (28\% del grupo de intervención y $54 \%$ del grupo control). Luego de la suplementación con harina de pescado no se encontró diferencia entre las mediciones de proteínas totales, albúmina e IMC en ambos grupos. Sin embargo, se observó un incremento en los niveles de hemoglobina $(p=0,003)$ y hematocrito $(p=0,002)$ en el grupo que recibió la harina, y solo la hemoglobina $(p=0,027)$ en el grupo control (Tabla 1). Al comparar los promedios de las diferencias (después-antes) no se encontraron diferencias entre los grupos ni para las pruebas de laboratorio ni para las pruebas de rendimiento físico (Tabla 2); cabe señalar, sin embargo, que en la prueba de Cooper ambos grupos recorrieron una mayor distancia al inicio que al final del estudio.

\section{DISCUSIÓN}

La harina de pescado empleada en este estudio está constituida en sus dos terceras partes por proteínas, por lo que se puede considerar como una fuente para cubrir la alta demanda energética de adolescentes deportistas. A pesar de ello, nuestro estudio no encontró que su consumo genere diferencias en el estado nutricional o condición física. Un aspecto importante a considerar con respecto a la ingesta de la proteína es que esta tiene mayor utilidad si es consumida después del ejercicio (10). Nuestros participantes recibieron la proteína durante el almuerzo previo a los entrenamientos, lo que pudo condicionar una menor asimilación y aprovechamiento.

Tabla 2. Promedios de las diferencias (después-antes) en los parámetros evaluados de adolescentes deportistas en complementación nutricional con harina de pescado.

\begin{tabular}{lcrc}
\hline & $\begin{array}{c}\text { Grupo de intervención } \\
\text { Media } \pm \mathrm{DE}\end{array}$ & $\begin{array}{c}\text { Grupo control } \\
\text { Media } \pm \mathrm{DE}\end{array}$ & $\boldsymbol{p}$ \\
\hline Proteínas totales $(\mathrm{g} / \mathrm{dL})$ & $0,1 \pm 0,4$ & $-0,1 \pm 0,4$ & 0,080 \\
Albúmina $(\mathrm{g} / \mathrm{dL})$ & $0 \pm 0,3$ & $0 \pm 0,4$ & 0,485 \\
Hemoglobina $(\mathrm{g} / \mathrm{dL})$ & $0,3 \pm 0,6$ & $0,3 \pm 0,7$ & 0,921 \\
Hematocrito $(\%)$ & $1,1 \pm 2,2$ & $0,5 \pm 2,5$ & 0,314 \\
$\mathrm{IMC}\left(\mathrm{kg} / \mathrm{m}^{2}\right)$ & $0,2 \pm 0,6$ & $0,1 \pm 1,0$ & 0,597 \\
Prueba de Cooper $(\mathrm{km})$ & $-0,2 \pm 0,3$ & $-0,2 \pm 0,4$ & 0,739 \\
\hline
\end{tabular}

DE: desviación estándar; IMC: índice de masa corporal. 
En la literatura no se encuentran estudios que evalúen el efecto de la proteína de pescado sobre la condición física, pero se tienen estudios que utilizan suplementos proteicos combinados con carbohidratos, con resultados distintos. Un grupo de futbolistas amateurs que consumió un suplemento mixto de carbohidratos y proteínas en un partido de fútbol simulado, consistente en 75 minutos de ejercicio intermitente, mejoró su capacidad para correr ${ }^{(11)}$. Asimismo, un grupo de ciclistas y triatletas femeninas que recibieron carbohidratos mezclados con proteínas mejoraron su rendimiento, en comparación a aquellas que solo recibieron carbohidratos ${ }^{(12)}$. Sin embargo, en otro estudio no se encontró mejoría en el desempeño de ejercicios de resistencia luego de la ingesta de $355 \mathrm{~mL}$ de bebidas con mezclas de carbohidratos y proteínas ${ }^{(13)}$. Estos resultados difieren con los nuestros, donde el desempeño de ambos grupos fue menor en la segunda evaluación. Esto probablemente se debió a la temporada en que realizaron las evaluaciones, pues los participantes estaban de vacaciones escolares durante la primera medición, mientras que en la segunda tenían que compartir la carga horaria escolar con las actividades deportivas, lo que podría haber disminuido el incentivo de los participantes. Esto se corrobora al no encontrar diferencia al comparar los promedios de las diferencias entre ambos grupos.

Llama la atención que los niveles de hemoglobina para ambos grupos y los niveles de hematocrito en el grupo de intervención se incrementaron. Yáñez et al. encontraron un aumento de la hemoglobina y del hematocrito en 12 niños entre 2 y 7 años alimentados con un concentrado de proteínas de pescado durante 3 meses (14). Pero al obtener, en esta investigación, un incremento en la hemoglobina en ambos grupos, incluso entre los que no recibieron la proteína, esta no sería la explicación. Los parámetros hematológicos pueden variar de acuerdo al tipo, intensidad y duración de los ejercicios ${ }^{(15)}$. Dos estudios encontraron que los niveles de hemoglobina y hematocrito se incrementaron en un grupo de adultos jóvenes sometidos a diferentes niveles de entrenamiento ${ }^{(16,17)}$, el continuo estrés al que se somete una persona cuando realiza ejercicios de forma habitual produce un incremento de hormonas e interleuquinas que estimulan la movilización de células progenitoras hematopoyéticas hacia la circulación ${ }^{(18,19)}$, lo cual podría explicar el presente hallazgo.

Este estudio presenta algunas limitaciones. La principal es que al tratarse de una muestra no probabilística no se pueden extrapolar los resultados encontrados. Otra limitación es la falta de cuantificación de los regímenes nutricionales de los participantes al inicio y durante el estudio, lo que no permite determinar el efecto de la proteína de anchoveta. Tampoco se consideró el efecto que podría tener el entrenamiento físico sobre los parámetros nutricionales, pudiendo ser esta una variable confusora no controlada. Finalmente, la pérdida de participantes para la segunda evaluación podría implicar un sesgo en los resultados.

En conclusión, en futbolistas adolescentes luego del consumo de proteína de pescado no hemos encontrado cambios en los parámetros de nutrición ni en su condición física. Los jugadores refirieron buena tolerancia a la proteína y no se observaron eventos adversos. Hubo un incremento de la hemoglobina en ambos grupos de estudio y solo del hematocrito en los que tomaron la proteína lo que puede ser consecuencia del entrenamiento físico.

Agradecimientos: a los doctores Yeny Bravo, Ana Mateo, Claudia Bernal, Julio Noda, Elizabeth Flores, Fabiola Gianella, Jacqueline Castañeda; y a los alumnos Veronica Salinas, María de los Ángeles Lazo, Claudia Alvizuri, Jessy Henriquez, Juan Diego Carballo, Priscilla Cavero, Albert Gonzales y Mario Jhonson de la Facultad de Medicina Alberto Hurtado de la Universidad Peruana Cayetano Heredia por su participación en la evaluación de los deportistas.

Contribuciones de autoría: RAT y LLO han participado en la concepción y diseño del artículo, análisis e interpretación de datos, redacción del artículo y aprobación de su versión final.

Fuentes de financiamiento: este estudio fue financiado por Pesquera Capricornio.

Conflictos de interés: los autores declaran no tener conflictos de interés en la publicación de este artículo.

\section{REFERENCIAS BIBLIOGRÁFICAS}

1. Spear B. Adolescent growth and development. J Am Diet Assoc 2002; 102 (3 Suppl): S23-9.

2. Rodriguez N, DiMarco N, Langley S; American Dietetic Association; Dietetians of Canada; American College of Sports Medicine. Position of the American Dietetic Association, Dietitians of
Canada, and the American College of Sports Medicine: Nutrition and athletic performance. J Am Diet Assoc 2009; 109(3): 509-27.

3. Food and Agriculture Organization of the United Nations [Internet]. Roma, Italia: Food and Agriculture Organization of the United Nations;
2004 [Citado el 10 de diciembre de 2012]. Human energy requirements. Disponible en: http://www.fao.org/ docrep/007/y5686e/y5686e00.htm

4. Instituto Nacional de Salud [Internet]. Lima, Perú: Instituto Nacional de Salud; 2012 [Citado el 10 de diciembre de 2012]. Requerimientos 
de energía para la población peruana. Disponible en: http://www.ins. gob.pe/repositorioaps/0/5/jer/$1 /$ Requerimiento $\% 20$ de $\% 20$ energ\%C3\%ADa\%20para\%20la\%20 poblaci\%C3\%B3n\%20peruana.pdf

5. Food and Agriculture Organization of the United Nations [Internet]. Roma, Italia: Food and Agriculture Organization of the United Nations; 1985 [Citado el 15 de diciembre de 2012]. Energy and protein requirements. Disponible en: http:// www.fao.org/docrep/003/AA040E/ AA040E00.htm\#TOC

6. Petrie H, Stover E, Horswill C. Nutritional concerns for the child and adolescent competitor. Nutrition 2004; 20 (7-8):620-31.

7. Boisseau N, Vermorel M, Rance M, Duché P, Patureau-Mirand P. Protein requirements in male adolescent soccer players. Eur J Appl Physiol 2007; 100(1): 27-33.

8. American Dietetic Association; Dietitians of Canada; American College of Sports Medicine. Position of the American Dietetic Association, Dietitians of Canada, and the American College of Sports Medicine: Nutrition and athletic performance. J Am Diet Assoc 2000; 100(12):1543-56.

9. Organización de las Naciones Unidas para la Agricultura y la Alimentación [Internet]. FAO; 20112012 [Citado: febrero 20132011]. Examen mundial de la pesca y la acuicultura 20122010 [11498 páginas]. Disponible en: http://www.fao.org/docrep/013/ i1820s/i1820s01.pdf http://www.fao. org/docrep/016/i2727s/i2727s01.pdf

10. Phillips S, Moore D, Tang J. A critical examination of dietary protein requirements, benefits, and excesses in athletes. Int J Sport Nutr Exerc Metab 2007; 17 Suppl:S58-76.

11. Alghannam A. Carbohydrate-protein ingestion improves subsequent running capacity towards the end of a footballspecific intermittent exercise. Appl Physiol Nutr Metab 2011; 36(5):748-57.

12. McCleave E, Ferguson-Stegall L, Ding Z, Doerner P, Wang B, Kammer L, et al. A low carbohydrate-protein supplement improves endurance performance in female athletes. J Strength Cond Res 2011; 25(4): 879-88.

13. Baty J, Hwang H, Ding Z, Bernard J, Wang B, Kwon B, et al. The effect of a carbohydrate and protein supplement on resistance exercise performance, hormonal response, and muscle damage. J Strength Cond Res 2007; 21(2):321-9.

14. Yáñez E, Ballester D, Maccioni A, Spada R, Barja I, Pak N, et al. Fishprotein concentrate and sunflower presscake meal as protein sources for human consumption. Am J Clin Nutr 1969; 22(7):878-86.

15. Banfi G, Lundby C, Robach P, Lippi G. Seasonal variations of haematological parameters in athletes. Eur J Appl Physiol 2011; 111(1):9-16.

16. Wardyn G, Rennard S, Brusnahan S, McGuire T, Carlson M, Smith L, et al. Effects of exercise on hematological parameters, circulating side population cells, and cytokines. Exp Hematol 2008; 36(2): 216-23.

17. Heisterberg M, Fahrenkrug J, Krustrup P, Storskov A, Kjær M, Andersen J. Extensive monitoring through multiple blood samples in professional soccer players. J Strength Cond Res 2012; [Epub ahead of print]

18. $\mathrm{Hu} \mathrm{M}$, Lin W. Effects of exercise training on red blood cell production: implications for anemia. Acta Haematol 2012; 127(3):156-64.

19. Bonsignore M, Morici G, Santoro A, Pagano M, Cascio L, Bonanno A, et al. Circulating hematopoietic progenitor cells in runners. J Appl Physiol 2002; 93(5):1691-7.

Correspondencia: Roberto Accinelli Tanaka. Dirección: Av. Honorio Delgado 430, Urb. Ingeniería, Lima 31, Perú.

Teléfono: (51) 998119480

Correo electrónico: raccinellit@yahoo.es

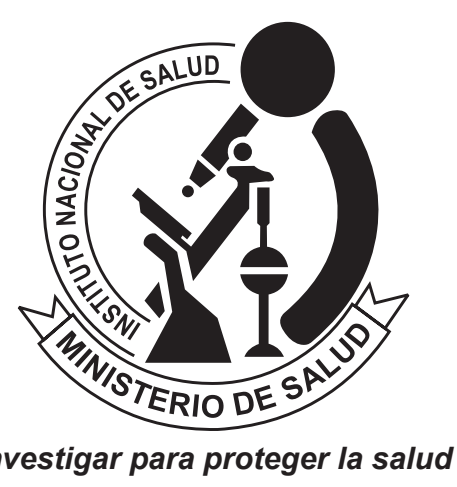

REVISTA PERUANA DE MEDICINA EXPERIMENTAL Y SALUD PÚBLICA CUMPLIENDO SUS METAS Y PROYECTÁNDOSE AL FUTURO

\section{Visite los contenidos de la revista en: www.ins.gob.pe/rpmesp}

